\title{
Littératies universitaires : Epistémologies, pratiques de l'écrit et expériences pédagogiques en interface dans l'enseignement supérieur
}

\author{
Cynthia Agra de Brito Neves ${ }^{1}$ \\ Universidade Estadual de Campinas - UNICAMP, Campinas, SP, Brésil
}

Fernanda Correa Silveira Galli ${ }^{2}$

Universidade Federal de Pernambuco - UFPE, Recife, PE, Brésil

Guillaume Nassau ${ }^{3}$

Université de Lorraine - UL, Nancy, France

\section{Présentation}

La perspective d'études des littératies universitaires a été, ces dernières années, le terrain de discussions fertiles, particulièrement dans le domaine de la linguistique appliquée, en témoignent les nombreuses recherches théoriques et pratiques développées au Brésil. Ces recherches ont permis de faire progresser le débat et les réflexions critiques sur les méthodes d'enseignement et d'apprentissage de la lecture et de l'écriture en langue maternelle et étrangère. Bien que le concept de littératie ait été pensé dans le contexte des études universitaires, il peut également être abordé dans d'autres contextes d'enseignement, car la lecture et l'écriture sont ici considérées comme des pratiques sociales (BARTON, 1994; GEE, 1996; STREET, 1984, 1995) qui diffèrent selon le contexte, la culture ou encore le sexe.

Ce dossier thématique intitulé "Littératies universitaires : Epistémologies, pratiques de l'écrit et expériences pédagogiques en interface dans l'enseignement supérieur" a pour objectifs, d'une part de permettre des réflexions épistémologiques sur l'écriture dans l'enseignement supérieur, et l'élargissement des possibilités de rapports et articulations

\footnotetext{
${ }^{1}$ Docteure en linguistique appliquée (Université d'Etat de Campinas - UNICAMP), Professeure au Département de linguistique appliquée (DLA), Institut d'études linguistiques (IEL), Université d'Etat de Campinas (UNICAMP). Orcid : https://orcid.org/0000-0002-5592-4409

Courriel : cynneves@unicamp.br ou cynthiaagrabneves@gmail.com

${ }^{2}$ Docteure en linguistique appliquée (Université d'Etat de Campinas - UNICAMP), Professeure au Département des Lettres du Centre pour la Communication et les Arts (CAC) de I'Université Fédérale du Pernambuco (UFPE). Orcid : https://orcid.org/0000-0002-4499-2908

Courriel : fcsgalli@hotmail.com

${ }^{3}$ Maître de conférence en sciences du langage, formateur à I'INSPE de Lorraine (Université de Lorraine), Nancy.

Courriel : guillaume.nassau@univ-lorraine.fr
} 
théoriques et méthodologiques dans le domaine des littératies universitaires. D'autre part, nous souhaitons soutenir des études susceptibles d'avoir un impact sur les dispositifs didactiques et les pratiques pédagogiques dans l'enseignement supérieur.

Ce dossier est également le résultat des activités menées dans le cadre plus large du projet Capes/Cofecub 834/15 (2015-2018), intitulé "Le discours universitaire dans la recherche et l'enseignement : questions autour de l'appropriation de la parole d'autrui", un partenariat entre des universités brésiliennes (PUC-Minas, UNESP-SJRP, UNICAMP et USP) et françaises (Université de Lorraine, Université Charles-de-Gaulle - Lille 3 et Université Grenoble Alpes). Le projet, qui a duré quatre années, a été coordonné par les professeures Juliana Alves Assis et Sophie Bailly.

Ce numéro thématique a été conçu à la fin de ces activités de recherche ${ }^{4}$, afin de diffuser les travaux des membres du projet et d'accueillir d'autres travaux sur le thème de la littératie universitaire. Par conséquent, ce volume comprend des articles qui traitent des pratiques d'écriture dans l'enseignement supérieur et se concentrent sur cinq axes thématiques, à savoir: 1) l'auteurité, 2) la relation à la parole d'autrui, 3) les dispositifs et ressources pédagogiques, 4 ) les systèmes de citation et 5 ) les genres discursifs.

Le premier axe est composé d'un article intitulé Autoria e internacionalização na escrita acadêmica: análise da principal organização profissional das engenharias elétrica e eletrônica, dans lequel Larissa Giacometti Paris et Rómina de Mello Laranjeira cherchent à identifier et à analyser, dans une perspective de recherche qualitative et interprétative, les conceptions de la rédaction académique de l'Institut de génie électrique et électronique (IEEE). Les auteurs analysent, dans des documents institutionnels, les articles scientifiques publiés par l'IEEE, en se concentrant sur deux conceptions impliquant des productions scientifiques : la création et l'internationalisation. De ce fait, ils montrent les relations de pouvoir institutionnel impliquées dans la définition de l'auteurité et la prédominance des compétences techniques dans les articles scientifiques rédigés en anglais, qui ne profitent qu'aux locuteurs natifs et mettent en échec le projet d'internationalisation de la science.

Le deuxième axe regroupe trois textes qui portent sur la relation à la parole d'autrui. Dans A dimensão interdiscursiva do dizer na escrita científica: o diálogo com a palavra de outrem em artigos científicos de jovens pesquisadores, José Cezinaldo Rocha Bessa examine les manières subtiles et complexes par lesquelles dix étudiants de master écrivent leurs textes académiques et scientifiques à partir des mots d'autrui. Dans une approche dialogique du langage caractéristique des théoriciens du cercle Bakhtine, Bessa conclut que le jeune chercheur, en s'appropriant l'écriture scientifique et en dialoguant de manière interdiscursive avec les mots des autres, est marqué par des pratiques de citation, à la fois en termes de forme et de contenu, qui ne sont pas considérées comme acceptables dans les conventions

\footnotetext{
${ }^{4}$ Lors du séminaire "Letramentos acadêmicos: epistemologias e práticas em interface", tenu à I'Université PUC-Minas, les 12 et 13 mars 2018, sous la coordination de la professeure Juliana Alves Assis.
} 
académiques et scientifiques.

L'observation de Bessa se retrouve dans la question posée par Sibely Oliveira Silva et Françoise Boch : "Comment construire le statut d'auteur quand on est étudiant ?", dans leur article Dialogar com o discurso de outrem na escrita acadêmica ou como construir uma posição de autor. Dans cet article, les auteurs analysent les textes académiques produits par des étudiants de Lettres afin d'identifier les procédures linguistico-textuelles, discursives et énonciatives qu'ils utilisent lorsqu'ils dialoguent avec le discours d'autrui dans leurs écrits académiques. Ils discutent des effets de ces choix - dont les normes sont parfois bien représentées dans les discours qui circulent à l'université, mais mal abordées dans les manuels d'écriture pour les étudiants - sur la construction de l'auteurité dans leurs textes.

Dans Discursos de orientadores brasileiros e franceses no e sobre ofeedback aos textos de seus mestrandos e doutorandos: um olhar sobre critérios e expedientes em torno da apropriação da palavra de outrem na escrita acadêmica, Juliana Alves Assis traite de I'utilisation du discours des autres dans les textes produits par les étudiants de troisième cycle des universités brésiliennes et françaises. Ancrée dans la perspective bakhtinienne, Assis réfléchit sur la fonction de la rétroaction écrite du professeur-guide par rapport aux textes de ses étudiants de master et de doctorat, et examine les points de vue de ces tuteurs - français et brésiliens - sur la façon dont les étudiants établissent la relation avec les mots des autres dans le processus de rédaction universitaire.

Le troisième axe est celui qui regroupe le plus grand nombre de recherches : douze articles au total, qui présentent des réflexions sur les dispositifs et ressources didactiques. $A$ interlocução com os pares na formação profissional e a construção da identidade do professorautor-formador, par Everton Vargas da Costa et Margarete Schlatter, est une recherche qui analyse la construction de l'identité de l'enseignant-formateur dans un contexte universitaire. Dans cette recherche ethnographique, les auteurs étudient les trajectoires, lors de formations (COSTA, 2013, 2018), de deux enseignantes de portugais comme langue supplémentaire (português como língua adicional) d'un cours de lettres dans une université fédérale du sud du Brésil. Pour Costa et Schlatter, c'est dans les interactions entre pairs - soit lorsqu'ils négocient la création d'une unité didactique, soit lorsqu'ils préparent la présentation des matériels dans un événement académique - que se construit leur identité professionnelle d'enseignant-formateur.

Dans Academic writing in higher education : focusing on courses of graduate programs of a public Brazilian University, Carlla Dall'Igna et Maria Ester Wollstein Moritz présentent les résultats de leurs recherches sur la façon dont la littératie de l'écriture académique est abordée dans seize domaines d'une université publique brésilienne. Dans cette cartographie, ils constatent que seulement $50 \%$ des programmes offrent des matières qui comprennent la rédaction universitaire, et que chaque matière aborde la littératie différemment. Un résultat alarmant qui amène les auteurs - et, par extension, nous amène aussi - à conclure que la littératie universitaire reste abordée de manière insuffisante dans les établissements brésiliens - d'où l'urgence d'inscrire ces discussions théoriques et pratiques à l'ordre du jour de la 
recherche, comme le propose ce dossier.

Des résultats tout aussi préoccupants se trouvent dans l'article Compreensões sobre $a$ formação para o/do ato de escrever na esfera acadêmica sob a perspectiva histórico-cultural, de Karoliny Correia et Aline Cassol Daga. Pour discuter des processus d'enseignement et de développement des sujets dans les genres de "l'acte d'écrire" de la sphère académique, les auteurs analysent, dans une perspective historico-culturelle basée sur les études bakhtiniennes et vygotskyennes, les utilisations de l'écriture dans une discipline de lecture et production textuelle (Leitura e Produção Textual) d'un cours de Lettres Portugaises, proposé dans le cadre de l'enseignement à distance, dans une université du sud du Brésil. Correia et Daga arrivent à une conclusion peu optimiste : la signature de l'acte semble être en marge du processus, dans un mouvement plus stationnaire que progressif, et les acteurs ne s'approprient pas efficacement les objets culturels impliqués dans les événements de la littératie académique.

Dans Enseñanza universitaria y literacidades académicas : perspectivas presentes y horizontes futuros, Virginia Orlando traite également des processus de lecture et d'écriture en milieu universitaire, plus précisément, l'auteur analyse trois "métaévénements" littéraires développés dans deux ateliers de lecture et d'écriture auquels elle a participé en tant qu'observateur-participant, dans une université publique à Montevideo en Uruguay. Les ateliers ont été proposés à des enseignants de différents domaines de cette institution et les "méta-événements" ont été analysés à partir de la "pédagogie de la multilittératie" (KALANTZIS et COPE, 2005). Les résultats témoignent de la nécessité de repenser ces pratiques (littéraires, discursives et sociales) et de proposer des actions didactiques plus efficaces impliquant la lecture et l'écriture dans les différents domaines disciplinaires d'une université.

Dans Letramento acadêmico e formação do professor de língua materna: um estudo de caso em um curso de letras, Márcia Adriana Dias Kraemer et Ramunielly Bonatti Longaretti étudient les enseignements académiques et scientifiques des étudiants en dernière phase de formation du cours de licence de lettres, portugais et espagnol, de l'Université fédérale de la Frontière Sud. Les résultats de l'analyse, fondés sur les hypothèses théoriques et empiriques concernant le développement des compétences en lecture et en écriture, sont prometteurs et démontrent que les sujets de la recherche présentent une participation périphérique légitime en tant qu'apprentis des pratiques de littératie impliquant l'enseignement, la recherche, et la vulgarisation. La pratique académique - avec des programmes qui privilégient, par exemple, les stages d'enseignement supervisés - permet l'insertion efficace de ces futurs enseignants dans des contextes éducatifs.

Les travaux de Camila Dilli, Bruna Morelo et Margarete Schlatter, intitulés $O$ ensino de leitura voltado a universitários indígenas: análise de uma unidade didática à luz dos estudos de letramento acadêmico, présentent un matériel didactique - en particulier un module sur les résumés - élaboré spécialement pour un cours dans une université fédérale du Brésil, dans le cadre d'un projet d'action positive pour la permanence des étudiants autochtones dans cette université jusqu'à l'obtention du diplôme. Les auteurs analysent comment les tâches de 
lecture et d'écriture proposées dans cette unité thématique, conçues pour les élèves des groupes prioritaires, soutenues par les Estudos de Letramento Acadêmico (ACLITS) et suivant les directives pédagogiques qui privilégient les pratiques sociales, sont valides, et rompent avec les directives historiques et les idéologies dominantes dans les pratiques d'enseignement universitaire.

Dans l'article $O$ gênero mapa mental e o letramento do professor de línguas, Luciane Sturm souligne que la formation universitaire des professeurs de langues est toujours un défi, car les étudiants ont peu de temps pour reconstruire leur identité et s'affirmer comme des enseignants autonomes. Consciente de ce défi, l'auteure argumente en faveur de l'utilisation de la carte mentale (BUZAN, 1995) comme outil d'apprentissage pour les étudiants tout au long de leur formation en littérature. L'auteure mise sur le potentiel des cartes mentales pour le développement de la cognition, de la créativité et de la littératie des élèves ; elle élabore ainsi une proposition pédagogique intéressante pour son utilisation.

Dalve Oliveira Batista-Santos étudie, dans 0 professor universitário como agente letrador: interfaces com o desenvolvimento do letramento acadêmico, la performance du professeur universitaire en tant qu'accompagnant au développement des littératies (KLEIMAN, 2006) dans la médiation et la gestion de différentes voix dans une activité dialogique de construction des sens. II s'agit d'une recherche interprétative qui a pour méthodologie la pensée à voix haute en groupe et qui est basée sur les hypothèses dialogiques du Cercle de Bakhtine et sur les nouvelles études de l'alphabétisation. Les données générées révèlent que les actions d'enseignement (réfuter, accepter et élargir les idées) permettent la construction de la culture académique : dans le cas analysé, l'enseignant-chercheur devient un agent d'enseignement par la médiation, la négociation et la gestion des voix des participants.

Incidencia de las trayectorias disciplinares en la escritura en el posgrado desde la perspectiva de los estudiantes, rédigé par María Isabel Pozzo, vise à décrire les effets des pratiques d'écriture dans les études supérieures - plus précisémment, en master à I'Universidad Tecnológica Nacional de Rosario, en Argentine - en partant du principe que ces pratiques sont différentes dans chaque discipline et/ou trajectoire scolaire. Le matériel d'analyse a été recueilli à partir des réponses des diplômés de troisième cycle aux questionnaires sur la perception des difficultés liées à la pratique de la rédaction dans différentes carrières, et les résultats indiquent qu'il y a peu ou pas de coïncidence entre les diplômés de la même discipline et que les trajectoires disciplinaires sont des phénomènes complexes.

L'article Experiências com a escrita na pós-graduação brasileira: uma proposta de diálogo com os letramentos acadêmicos, de Guilherme Brambila, suit le thème de cet axe en discutant des places de l'écriture académique suite à l'obtention d'un master ou d'un doctorat au Brésil. Cette fois, l'analyse est centrée sur les posts des étudiants de troisième cycle du groupe "Capes Scholars" sur le réseau social Facebook, un espace dans lequel ils rapportent leurs expériences douloureuses lors de la production de textes du genre académique. Dans cette recherche, Brambila sélectionne comme corpus trois posts où les étudiants confessent 
leurs difficultés pour rédiger leurs mémoires. Cette étude est guidée par les hypothèses de l'enseignement académique dans le contexte brésilien (FISCHER, 2010 ; FIAD, 2011) et l'analyse suit le paradigme indicatif sur le plan méthodologique (GINZBURG, 1986).

Dans le texte Da dimensão sociocomunicativa à arquitetura textual na abordagem didática do gênero resenha acadêmica, Regina Celi Mendes Pereira et Evandro Gonçalves Leite présentent une expérience didactique des activités de production - d'écriture et de réécriture - de la revue universitaire avec des étudiants en littérature. Ancrés dans les contributions théoriques et méthodologiques de l'interactionnisme sociodiscursif affilié à la perspective des Lettres Académiques, les auteurs concluent que, dans le processus de rédaction du genre revue, il y a un entrelacement d'aspects sociocommunicatifs, discursifs et linguistiques. Ils soulignent également que la rédaction académique est une activité qui implique des questions qui vont au-delà du matérialisme textuel, et exigent expérience et agençabilité, car ce sont ces pratiques (sociales) qui peuvent garantir de meilleures conditions d'appropriation des genres aux étudiants.

Pour finaliser cet axe, Cynthia Agra de Brito Neves présente, dans Les genres académiques au ProFIS : un rapport d'expérience et d'inclusion sociale à I'Unicamp, le Programme interdisciplinaire d'enseignement supérieur de I'Unicamp (ProFIS), un cours pilote créé en 2011 et offert aux étudiants des écoles publiques à Campinas. Dans cet article, l'auteure fait part de son expérience d'enseignement, en 2017, dans le domaine de la lecture et de la production de textes académiques, qu'elle a enseigné aux étudiants du ProFIS. Neves analyse la production des textes académiques de ces étudiants, tels que la rédaction de la revue critique et de l'article scientifique, et les séminaires oraux sur le genre, soulignant dans ces activités de littératie, les succès et les difficultés d'enseignement et d'apprentissage de ces genres académiques.

Le quatrième axe consiste en un article sur les systèmes de citation. Discurso acadêmico: a regulação do poder disciplinar e do panóptico, par Beatriz Gil et Fernanda Correa Silveira Galli, présente une réflexion sur l'opérationnalisation du pouvoir disciplinaire dans les pratiques qui entourent le discours académique à notre époque. Sur la base de la méthodologie généalogique foucaldienne, les auteurs analysent les systèmes métriques académiques et scientifiques de la base de données Google Academic et de l'index $\mathrm{H}$, qui sont considérés comme des marqueurs académiques de la productivité. Comme résultats, Gil et Galli soulignent les principes de visibilité et d'invariabilité du travail panoptique comme outils disciplinaires dans le contexte académique, ce qui conduit les chercheurs à se transformer inconsciemment en simples témoins de la production scientifique et, par conséquent, à s'adapter à la normalité déterminée par les institutions.

Le cinquième et dernier axe regroupe cinq articles qui traitent du thème des genres de discours. A produção escrita de artigo no contexto acadêmico: as contribuições da abordagem sistêmico-funcional, par Záira Bomfante dos Santos et Flaviane Faria Carvalho, s'intéresse à la façon dont l'approche linguistique systémique-fonctionnelle, en dialogue avec la sociorhétorique, peut être appliquée dans la production des articles scientifiques produits par les 
étudiants dans le domaine des sciences humaines et naturelles et des mathématiques. Comme résultats, les auteurs : (i) soulignent que la citation est la stratégie rhétorique la plus importante dans les articles, dans les deux domaines, et (ii) que les sciences humaines ont tendance à accorder moins d'importance aux résultats de la recherche que les sciences de la nature et des mathématiques, qui, à leur tour, valorisent les impacts et l'application des résultats à la société.

Dans son article intitulé Entre buscar contribuir e la contribuition: a modalização em resumos científicos em português e francês, Sandra Dias Loguercio rend compte d'une étude comparative - onomasiologique et semasiologique - sur les marques de modulation présentes dans les résumés scientifiques produits en portugais et en français, afin de mieux caractériser ce genre. Comme résultats, Loguercio souligne que la langue utilisée dans les résumés confirme la prépondérance d'une posture scientifique et, en même temps, démontre que les résumés en portugais sont caractérisés par la posture d'intention (volitive), tandis que les résumés en français sont configurés par la posture d'affirmation de soi (évaluative). Pour l'auteur, ces aspects sont importants et doivent être pris en compte dans la pédagogie des langues et de la traduction.

Dans Les entrées en matière non théoriques dans des mémoires de master et des articles de revues, Bertrand Daunay et Daniel Bart comparent les introductions de mémoires de master et d'articles de professeurs docteurs - dans le domaine de la didactique, en langue française - et décrivent les spécificités du discours de ce genre universitaire. Dans l'enquête, les auteurs cherchent à discuter du rôle des références faites par les chercheurs à d'autres domaines que la théorie, qu'ils considèrent comme un défi dans le processus d'apprentissage de l'écriture académique. A la suite de l'analyse exploratoire, Daunay et Bart cartographient les convergences formelles des références non théoriques présentes dans les textes analysés - des enseignants et des élèves - ainsi que les différences liées au type de référence utilisé.

Dans I'article Letramento acadêmico: dimensões escondidas em rasuras em contexto digital, Tatiane Henrique Sousa Machado, Cristiane Carneiro Capristano et Neiva Maria Jung, analysent les ratures dans des articles d'opinion écrits par des universitaires, en contexte digital. Partant d'une vision énonciative-discursive de la langue, les auteures concluent que les ratures indiquent quatre négociations régulières (cf. AUTHIER-REVUZ, 1998), qui concernent le choix du lexique, la norme linguistique, la définition du genre et les positions exprimées dans les textes. Ces négociations apparaissent dans le genre article d'opinion soit montrées (mais opaques), soit cachées (non explicites), et sont mises en évidence dans la réflexion comme les effets de la pluralité des significations des relations de pouvoir instituées et réaffirmées dans les pratiques de littératie.

Dans Letramentos acadêmicos em PLE: escrevendo a sociedade e a cultura brasileira na Coreia do Sul, Denis Leandro Francisco présente une discussion sur la pratique de l'écriture avec des diplômés des études brésiliennes dans le contexte de l'enseignement supérieur sudcoréen. S'appuyant sur le modèle idéologique de la littératie (STREET, 1995), l'auteur aborde l'écriture académique - du genre portfolio - comme un outil didactique qui peut développer à 
la fois l'autonomie et le positionnement critique des étudiants. En guise de conclusion, Francisco souligne, d'une part, les défis rencontrés dans le contexte coréen analysé, qui ont trait à la méconnaissance par les étudiants des pratiques de littératie académique, en particulier le genre du portfolio ; d'autre part, il évalue cette "pratique pédagogique" de la production écrite comme pertinente pour l'apprentissage de la langue-culture cible des étudiants dans le contexte universitaire.

Tous les articles sélectionnés pour ce numéro thématique présentent des recherches, à la fois brésiliennes et étrangères, qui pourraient intégrer plus d'un axe, parce qu'elles ont été pensées de manière poreuse et que les travaux qui y sont contemplés dialoguent entre eux au sens de Bakhtine. De plus, le présent dossier se distingue par la quantité, la qualité et la diversité des articles soumis, ce qui prouve une fois de plus la pertinence du thème et son urgence dans le monde contemporain. Pour finir, nous aimerions remercier les auteurs du Brésil, d'Argentine, de France et d'Uruguay, qui ont contribué par leurs travaux à l'approfondissement de la réflexion sur les littératies universitaires.

\section{Références}

AUTHIER-REVUZ, J. Palavras incertas: as não coincidências do dizer. Campinas: Editora da UNICAMP, 1998.

BARTON, D. Literacy: an introduction to the ecology of written language. London: Blackwell, 1994.

BUZAN, T. The mind map book. 2. ed. London: BBC Books, 1995.

COSTA, E. V. Práticas de formação de professores de português língua adicional em um Instituto Cultural Brasileiro no exterior. Dissertação (Mestrado em Letras). Porto Alegre: Universidade Federal do Rio Grande do Sul, 2013.

COSTA, E. V. Eventos de formação de professores de português como língua adicional: a organização das práticas e as trajetórias de participação em um estudo interpretativo sobre aprender a ensinar. Tese (Doutorado em Letras). Porto Alegre: Universidade Federal do Rio Grande do Sul, 2018.

FIAD, R. S. A escrita na universidade. Revista da ABRALIN, v. Eletrônico, n. Especial, p. 357-369, 2a parte, 2011.

FISCHER, A. Os usos da língua na construção de sujeitos letrados: relações entre a esfera escolar e a acadêmica. Acta Scientiarum. Language and Culture, Maringá, v. 32, n. 2, p. $215-$ 224, 2010.

GEE, J. P. Social linguistics and literacies: ideology in discourses. London: Falmer, 1996.

GINZBURG, C. Mitos, Emblemas, Sinais: morfologia e história. São Paulo: Companhia das Letras, 1986. 
KALANTZIS, M.; COPE, B. A multiliteracies pedagogy: a pedagogical supplement. In: KALANTZIS, M.; COPE, B. (Eds.) Multiliteracies: literacy learning and the design of social futures. London and New York: Routledge, 2005. p. 239-248.

KLEIMAN, A. B. Processos identitários na formação profissional - O professor como agente de Letramento. In: CORRÊA, M.; BOCH, F. Ensino de língua: representação e letramento. Campinas, SP. Mercado das Letras, 2006.

STREET, B. V. Literacy in theory and practice. Cambridge: Cambridge University Press, 1984.

STREET, B. V. Social literacies: critical approaches to literacy development, ethnography and education. London: Longman, 1995. 